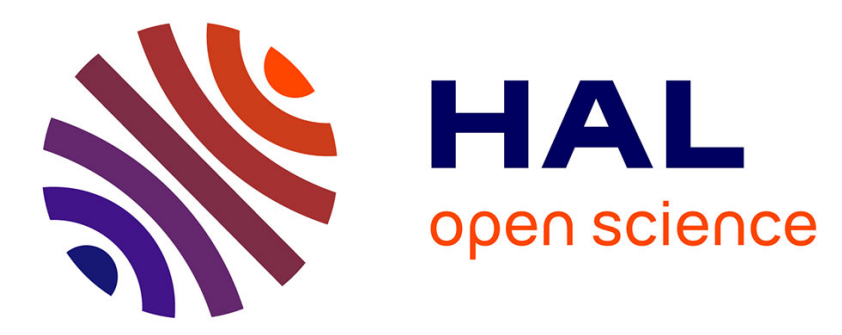

\title{
Analyses polliniques et parasitologiques préliminaires de coprolithes de carnivores du site moustérien des Ramandils (Port-la-Nouvelle, Aude, France)
}

Anne-sophie Lartigot-campin, Hélène H. Moné

\section{- To cite this version:}

Anne-sophie Lartigot-campin, Hélène H. Moné. Analyses polliniques et parasitologiques préliminaires de coprolithes de carnivores du site moustérien des Ramandils (Port-la-Nouvelle, Aude, France). Quaternaire, 2017, vol. 28/2, pp.217 - 224. 10.4000/quaternaire.8130 . hal-01598408

\author{
HAL Id: hal-01598408 \\ https://hal.science/hal-01598408
}

Submitted on 2 Dec 2020

HAL is a multi-disciplinary open access archive for the deposit and dissemination of scientific research documents, whether they are published or not. The documents may come from teaching and research institutions in France or abroad, or from public or private research centers.
L'archive ouverte pluridisciplinaire HAL, est destinée au dépôt et à la diffusion de documents scientifiques de niveau recherche, publiés ou non, émanant des établissements d'enseignement et de recherche français ou étrangers, des laboratoires publics ou privés. 


\section{Quaternaire}

Revue de l'Association française pour l'étude du

Quaternaire

vol. 28/2 | 2017

Volume 28 Numéro 2

\section{Analyses polliniques et parasitologiques préliminaires de coprolithes de carnivores du site moustérien des Ramandils (Port-la-Nouvelle, Aude, France)}

Preliminary palynological and parasitological analyses of carnivora coprolites from the mousterian site of les ramandils (port-la-nouvelle, aude, france)

\section{Anne-Sophie Lartigot-Campin and Hélène Mone}

\section{revues.org}

Electronic version

URL: http://quaternaire.revues.org/8130

DOI: 10.4000/quaternaire.8130

ISSN: 1965-0795
Publisher

Association française pour l'étude du quaternaire

Printed version

Date of publication: 29 May 2017

Number of pages: $217-224$

ISSN: $1142-2904$

Electronic reference

Anne-Sophie Lartigot-Campin and Hélène Mone, « Analyses polliniques et parasitologiques préliminaires de coprolithes de carnivores du site moustérien des Ramandils (Port-la-Nouvelle, Aude, France) », Quaternaire [Online], vol. 28/2 | 2017, Online since 01 June 2017, connection on 13 July 2017. URL : http://quaternaire.revues.org/8130 ; DOI : 10.4000/quaternaire.8130 


\title{
ANALYSES POLLINIQUES ET PARASITOLOGIQUES PRÉLIMINAIRES DE COPROLITHES DE CARNIVORES DU SITE MOUSTÉRIEN DES RAMANDILS (PORT-LA-NOUVELLE, AUDE, FRANCE)
}

\author{
Anne-Sophie LARTIGOT-CAMPIN ${ }^{1} \&$ Hélène MONE ${ }^{2}$
}

\begin{abstract}
RÉSUMÉ
Les fouilles du site des Ramandils ont livré une faune abondante associée à une industrie moustérienne, mais le remplissage sédimentaire n'a pas été favorable aux analyses polliniques. La découverte de nombreux coprolithes offre l'opportunité de réaliser une nouvelle analyse pollinique pour obtenir des données sur la composition du paysage végétal, visité par les prédateurs (carnivores et hommes) et contemporain du site. Ces tests mettent en évidence la présence d'ascaridioses, provoquées par Toxocara sp. et Ascaris sp., qui n'avaient pas encore été diagnostiquées, à cette époque du Pléistocène supérieur dans le Sud de la France.
\end{abstract}

Mots-clé : coprolithes, palynologie, parasitologie, paléoenvironnement, Pléistocène supérieur

\section{ABSTRACT}

PRELIMINARY PALYNOLOGICAL AND PARASITOLOGICAL ANALYSES OF CARNIVORA COPROLITES FROM THE MOUSTERIAN SITE OF LES RAMANDILS (PORT-LA-NOUVELLE, AUDE, FRANCE)

Excavations at the Ramandils site have yielded abundant faunal remains associated with a Mousterian industry, but the infilling was not favorable to pollen analysis. The discovery of numerous coprolites offers the opportunity to carry out a new pollen analysis to obtain data on the composition of the plant landscape, visited by the predators (carnivores and humans) and contemporary of the site. These tests reveal the presence of ascaridiasis, caused by Toxocara and Ascaris, which had not yet been diagnosed, during this period of the Upper Pleistocene in the South of France.

Key-words: coprolites, palynology, parasitology, palaeoenvironment, Upper Pleistocene

\section{1 - INTÉRÊTS DES ANALYSES POLLINIQUE ET PARASITOLOGIQUE DES COPROLITHES}

Les coprolithes, du grec kopros (excrément) et lithos (pierre) (Buckland, 1829) sont des excréments fossilisés, c'est-à-dire desséchés ou minéralisés, produits par des invertébrés ou des vertébrés, marins ou terrestres. Dans les sites archéologiques, leur mise en évidence témoigne de la présence de l'animal émetteur à un moment précis, les coprolithes de carnivores (canidés, félidés) et en particulier ceux de broyeurs d'os (hyénidés) étant rencontrés plus fréquemment. En effet, les fragments d'os servent de charpente à l'excrément qui garde ainsi sa forme et le broyat implique un fort taux de minéralisation et une richesse en phosphates importante, favorisant une fossilisation rapide (Martill, 1994). Les coprolithes solides ne sont pas détruits par les insectes coprophages et leur conservation est possible en surface, dans un environne- ment sec ou en grotte (Larkin et al., 2000). Ces particularités favorisent la conservation du contenu du coprolithe, dont la nature est influencée directement, ou indirectement, par le comportement de l'animal émetteur. Il s'agit aussi bien de macro-restes (os broyés, poils, coquilles, graviers, graines, débris végétaux par exemple) que de micro-restes (pollen, phytolithes, œufs d'endoparasites) ou de l'ADN.

L'analyse palynologique de coprolithes a été initiée dans les années 1960 (Martin \& Sharrock, 1964) et constitue une base fiable pour la reconstitution environnementale (Leroi-Gourhan, 1966 ; Gonzales-Samperiz et al., 2003 ; Fugassa et al., 2009 ; Gatta et al., 2016, par exemple), les coprolithes de hyènes ayant un potentiel de conservation des palynomorphes supérieur aux sédiments détritiques (Scott, 1987 ; Scott \& Brink, 1992 ; Carriòn et al., 2000 ; Scott et al., 2003, par exemple). Elle complète les données polliniques issues du remplissage ou palie aux

\footnotetext{
${ }^{1}$ Université de Perpignan Via Domitia UPVD - EPCC CERP Tautavel UMR 7194 CNRS-UPVD-MNHN (HNHP), avenue Léon-Jean Grégory - FR-66720 TAUTAVEL. Courriel : aslc@cerptautavel.com

${ }^{2}$ Université de Perpignan Via Domitia UPVD - Laboratoire Interactions Hôtes-Parasites-Environnements (IHPE) UMR 5244 CNRS-UPVDUM-IFREMER, 58 avenue Paul Alduy, FR-66860 PERPIGNAN. Courriel : mone@univ-perp.fr
} 
manques de données (Argant \& Philippe, 2011 ; Argant $\&$ Bonifay, 2011, par exemple).

Cette approche environnementale peut être complétée par une étude parasitologique, qui consiste à déterminer les restes de fossiles parasites dans les sites archéologiques (Bouchet, 2001). Les helminthes endoparasites ont des cycles de vie bien spécifiques, parasitant un hôte préférentiel appelé " hôte définitif » et, suivant les cycles, en ayant recourt à des hôtes intermédiaires. Une fois installés dans l'organe-cible de leur hôte définitif, les vers se reproduisent et pondent des œufs, qui sont alors libérés dans l'environnement extérieur, dans les urines ou les selles de leurs hôtes. Les excréments fossilisent en emprisonnant notamment les grains de pollen et les œufs qui résistent aux agressions de nature physico-chimique du milieu environnant (Wharton, 1980 ; Bouchet \& Boulard, 1991). La détermination de ces œufs permet d'identifier les parasitoses contemporaines de l'époque étudiée, mais également de reconnaître la présence du ou des hôtes du parasite dans l'environnement du site (Lebailly et al., 2003 ; Harter et al., 2005).

Les multiples campagnes de fouilles, menées au Ramandils de 1989 à 1994 par Paul Boutié, ont permis de mettre au jour quelque 155 coprolithes, répartis sur la totalité de la séquence sédimentaire continentale. Leur analyse pollinique pourrait palier aux manques de données sur l'environnement végétal car celles menées sur le remplissage avaient livré des résultats difficilement exploitables (Renault-Miskovsky, 1972 ; Messouci, 1993). Les analyses polliniques et parasitologiques, réalisées conjointement sur les mêmes coprolithes devraient permettre de préciser le contexte environnemental de cette période du Pléistocène supérieur.

\section{2 - LE SITE DES RAMANDILS}

Le site des Ramandils (Port-la-Nouvelle, Aude, France) s'ouvre dans un promontoire calcaire, le cap Romarin, à 1,50 mètre d'altitude et à $1500 \mathrm{~m}$ du rivage actuel de la mer Méditerranée (fig 1). Son remplissage, d'une puissance de 6 mètres, se compose d'une plage marine daté de $128000 \pm 15000$ ans B.P., par ESR (Yokoyama et al., 1987), sur laquelle reposent 5 ensembles stratigraphiques continentaux à la base desquels s'écoule une source (fig. 2).
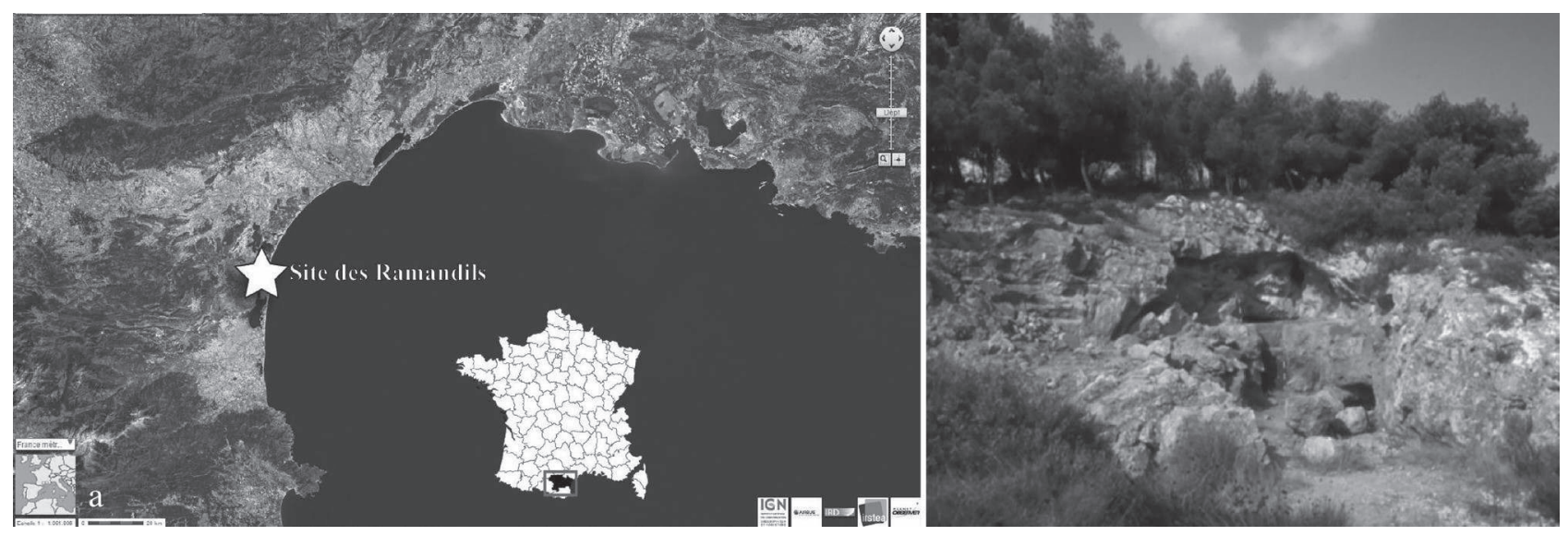

Fig. 1 : Situation géographique (d'après Géoportail) et vue du site des Ramandils (P. Boutié).

Fig. 1: Geographic context (after Géoportail) and view of the site (P. Boutié).

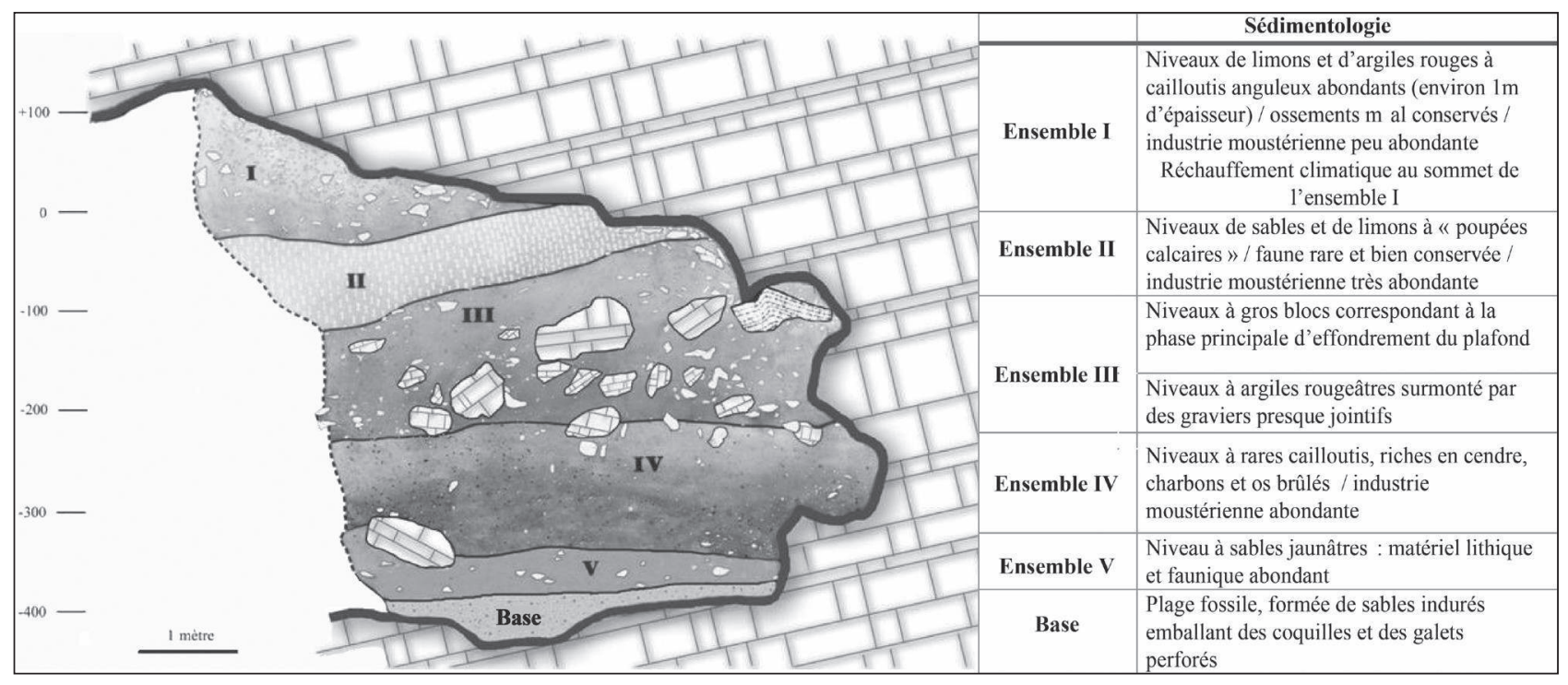

Fig. 2 : Stratigraphie synthétique du site (Lumley de, in Boutié, 1984).

Fig. 2: Synthetic stratigraphy of the site (Lumley de, in Boutié, 1984). 
Les nombreuses campagnes de fouilles ont permis de mettre au jour deux dents humaines appartenant vraisemblablement à Homo neanderthalensis, associées à une industrie moustérienne abondante (Molès, 2008) et à de nombreux ossements d'herbivores (Cervus, Dama, Equus germanicus, E. hydrunthinus, Sus, Bos primigenius) et de carnivores, comme le lynx, la hyène ou le loup, le renard et le blaireau (Boutié et al., 1994 ; Banes \& Dorigny, 2005 ; Rusch, 2015 ; Boulbes \& Rusch, 2016).

Les précédentes analyses polliniques ont apporté des résultats ponctuels et taxonomiquement peu diversifiés dans les ensembles II et V. Le paysage dans ces deux ensembles se composait d'une vaste steppe à graminées, ponctuées de bosquets de pins et de taxons thermophiles. Les auteurs proposent la présence d'un climat froid et sec, adouci par la présence de la mer dans les deux ensembles. Le reste du remplissage détritique (ensembles IV, III et I) et la stalagmite surplombant le remplissage, n'ont pas donné de résultats exploitables (Renault-Miskovsky, 1972 ; Messouci, 1993).

\section{3 - MATÉRIEL ET MÉTHODES}

Pour cette étude préliminaire, des analyses polliniques ont été menées sur 12 coprolithes, découverts dans les ensembles I, II, IV et V. Ceux de l'ensemble III n'ont pas encore été étudiés. Le choix s'est porté sur les coprolithes les plus gros (entre 6 et 142 grammes ; fig. 3).

Les analyses palynologiques et parasitologiques nécessitent de respecter des étapes communes, en début de procédure. Comme les deux protocoles spécifiques détruisent le matériel étudié, il faut les photographier pour les archiver et les décrire rigoureusement (forme, poids, taille, volume, présence d'inclusions et de traces liées à l'enfouissement et à la conservation in situ et aux processus post-dépositionnels, couleur interne et externe), ce qui permet de les identifier (Vivent, 1989 ; Jouy-Avantin, 1997, 2004 ; Jouy-Avantin et al., 1999, 2003).
Les coprolithes sont ensuite soigneusement brossés sous un filet d'eau distillée, afin de limiter au mieux les risques de pollution et divisés en deux parties. Le traitement classique de l'extraction sporo-pollinique est appliqué à chaque première fraction de coprolithe (Argant, 2010, par exemple), les autres parties subissant celui de l'analyse parasitologique (Van Cleave \& Ross, 1947 ; Callen \& Cameron, 1960).

\section{4 - RÉSULTATS}

Au regard de la couleur beige, de la forme arrondie, de la richesse en carbonates (broyat d'os), des dimensions (fig. 3) et de la composition du cortège des carnivores, les coprolithes issus des ensembles I, II et V peuvent être attribués à Crocuta spelea. Les coprolithes de plus petites tailles des ensembles I et IV pourraient appartenir à Lynx sp. ou à Canis lupus.

\section{1 - ANALYSE PALYNOLOGIQUE}

L'ensemble du culot a été observé pour chaque échantillon. Sept coprolithes sont très pauvres en pollen, voire stériles, avec des sommes de base comprises entre 3 et 44 grains de pollen, répartis entre 1 et 14 taxons (tab. 1). Les cinq coprolithes qui ont livré la plus grande quantité de pollen se situent dans les ensembles stratigraphiques, I, II et V. Ils ont des sommes de base comprises entre 184 et 1130 pollens, pour des concentrations sporo-polliniques faibles, comprises entre 19 et 71 grains par gramme de coprolithe. La diversité taxonomique est constante (entre 31 et 35 taxons) et le seuil de 20 taxons polliniques, exigé pour l'interprétation des données, est ici largement dépassé. Bien que les grains de pollen soient moins abondants dans ces supports d'analyse, ils y sont relativement bien identifiables. Les taux de Varia (entre 3 et $29 \%$ ), sont courants dans les analyses de coprolithes (Yll et al., 2006 ; Carriòn et al., 2007 ; Gatta et al., 2016). Ils sont dus à la présence de grains de pollen minéralisés ou

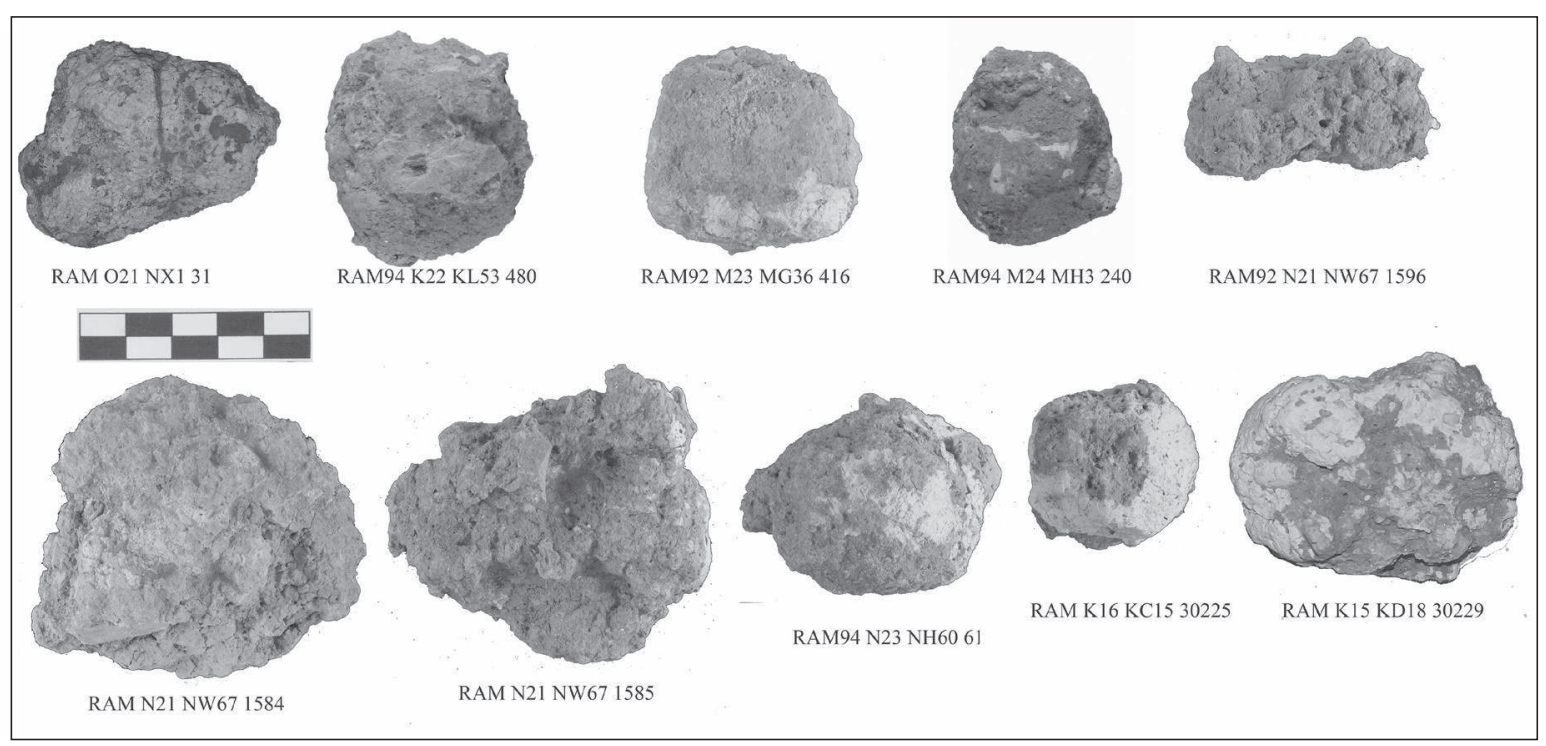

Fig. 3 : Coprolithes entiers étudiés.

Fig. 3: Whole coprolites studied. 


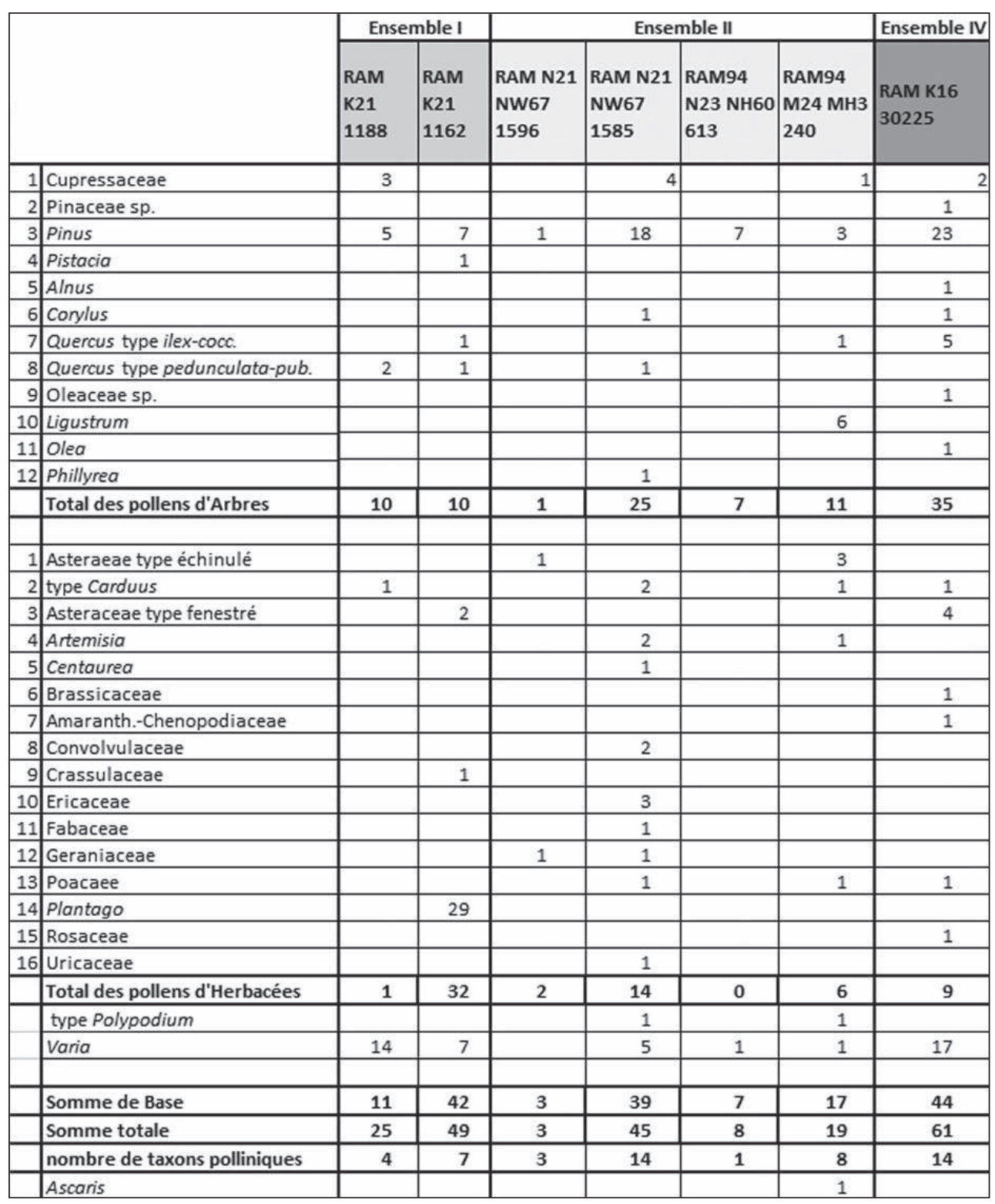

Tab. 1 : Inventaire des données polliniques issues des coprolithes stériles

Tab. 1: Inventory of the pollen data resulting from coprolites too poor in pollen.

collés à de la matière organique et pliés, rendant difficile l'attribution à un taxon spécifique. C'est notamment le cas des Pinaceae des coprolithes de l'ensemble II.

L'ensemble des données valables obtenues révèle l'image d'un paysage relativement ouvert (taux de pollen d'herbacées compris entre 35 et $86 \%$ ), avec une grande diversité des herbacées (41 taxons au total), et une dominance constante des Cichorioideae (entre 6 et $25 \%$ ) et des Asteroideae (entre 2 et $29 \%$ ), suivies des Poaceae (entre 2 et $4 \%$ ). Le cortège du coprolithe RAM O21 NX1 31 de l'ensemble I est marqué par une sur-représentation de Carduus (67\%).

Les arbres (28 taxons au total) sont dominés par Pinus sp. (entre 11 et $45 \%$ ). De nombreux arbres mésophiles et thermophiles sont présents, mais toujours dans de très faibles pourcentages (toujours inférieurs à $5 \%$ ). Les éléments caducifoliés, comme Quercus type pedunculuata-pubescens, Fraxinus, Sambucus-Viburnum, Betula, Corylus, Acer et Ulmus, sont sporadiquement présents dans les échantillons, tandis que les sempervirens tels que les Cupressaceae (jusque $8 \%$ ) et Quercus type ilex (jusque $3 \%$ ) sont plus abondants. La ripisylve est enregistrée ponctuellement, avec Alnus et Salix (fig. 4).

\section{2 - ANALYSE PARASITOLOGIQUE}

Les observations parasitologiques ont livré 2 variétés d'œufs de vers parasites, d'environ $70 \mu \mathrm{m}$. Le premier se caractérise par une enveloppe épaisse et rugueuse, avec une ornementation en filet (fig. 5a), le second montre une coque épaisse mamelonnée (fig. 5b). Il s'agit d'œufs de vers endoparasites de la classe des nématodes, de l'ordre des Ascaridida et des genres Toxocara sp. (famille des Toxocaridae) et Ascaris sp. (famille des Ascarididae).

Ces œufs ont été trouvés dans trois coprolithes différents, appartenant tous à l'ensemble stratigraphique II : deux œufs d'Ascaris sp. et quatre œufs de Toxocara sp. ont été identifiés dans le coprolithe "RAM92 M23 


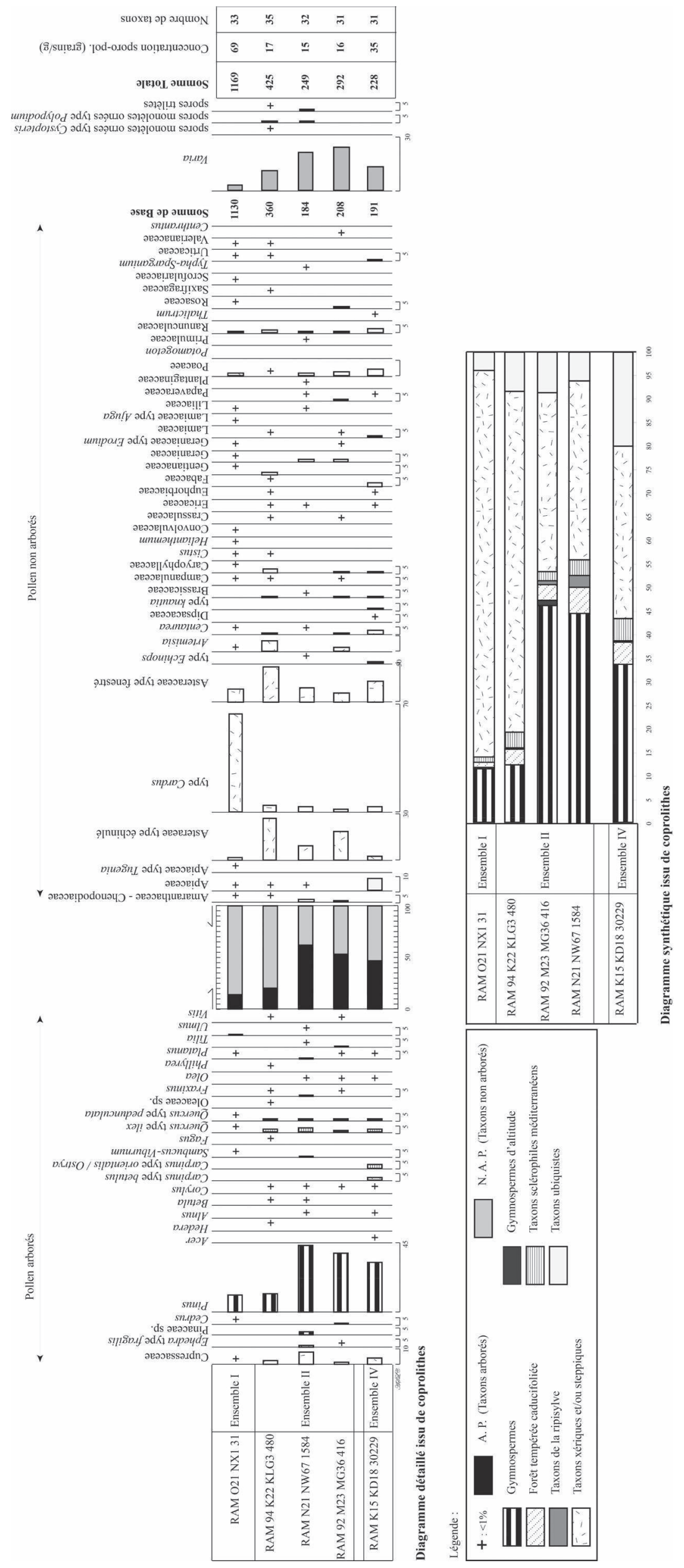




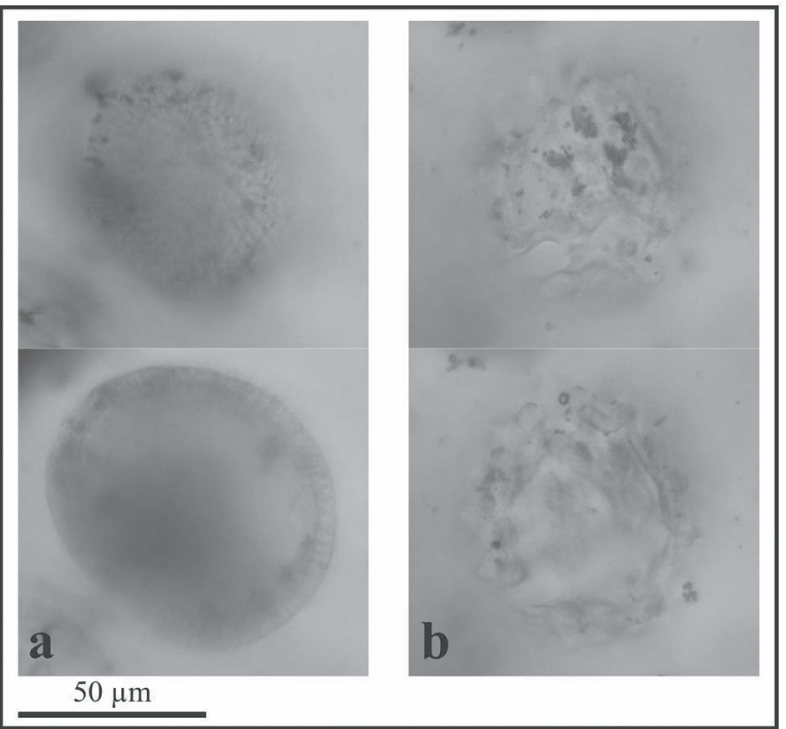

Fig. 5 : Cufs de Toxocara sp. (a) et d'Ascaris sp. (b) (RAM92 M23 MG36 416).

Fig. 5: Toxocara sp. (a) and Ascaris sp. (b) eggs (RAM92 M23 $M G 36$ 416).

MG36 $416 »$, cinq œufs d'Ascaris sp. et un œuf de Toxocara sp. ont été découverts dans le coprolithe « RAM94 K22 KLG53 480 ». Enfin, un seul œuf d'Ascaris a été reconnu dans le coprolithe « RAM94 M24 MH3 240 ».

\section{5 - INTERPRÉTATIONS DES RÉSULTATS}

\section{1 - DONNÉES PALYNOLOGIQUES}

Un carnivore ingère le pollen quotidiennement, en buvant, en respirant, en se léchant et en mangeant la peau, la panse ou les viscères des herbivores, ou même de la terre, de l'herbe et des graviers. L'ensemble de ces grains de pollen provient des végétaux en fleurs rencontrés par l'animal ou ses proies et reflète donc l'environnement dans lequel il évolue, dans un périmètre assez restreint, local ou régional, et peut varier selon la saison (Argant, 1990). Les quantités de pollen obtenues dans cette étude sont évidemment faibles au regard de celles extraites des tourbes ou argiles organiques. Cela n'a rien de surprenant au regard des résultats obtenus dans le cadre d'analyses similaires (Argant \& Bonifay, 2011). Mais cela est compensé par la garantie que le matériel sporo-pollinique soit contemporain de l'animal-émetteur et représente une période d'enregistrement extrêmement courte, le transit intestinal se réalisant en 24 à 36 heures. Il ne faut d'ailleurs pas écarter les circonstances d'absorption du pollen ni le rôle de la saison dans l'apport pollinique, qui pourraient expliquer les concentrations polliniques très basses par rapport aux taux couramment rencontrés dans des coprolithes du Pléistocène moyen et supérieur (Carriòn et al., 2007, par exemple).

La forte proportion de Pinus sp. laisse envisager la présence de ce taxon à proximité du site. Parmi les taxons arborés, de nombreux genres sont héliophiles, comme par exemple Pinus, Quercus, les Cupressaceae, Fraxinus ou Betula. Leur diversité évoque une formation boisée peu dense, probablement sous forme de bosquets. La présence de sols constamment humides, liés à la présence d'une source comme celle qui coule encore de nos jours à la base du remplissage, est évoquée par Alnus et Salix ou encore par Fraxinus, ou encore par les Renonculaceae, présentes dans l'ensemble des échantillons, dont certaines espèces affectionnent les zones humides. Cette hypothèse est confirmée par la détermination de quelques coquilles de Pseudoschizae, dans les coprolithes O21 NXI 31 et RAM 94 M23 MG36 416.

Certaines Asteraceae, comme Carduus, et les Cichorioideae sont nitrophiles (Florenzano et al., 2015). Elles se développent sur des sols enrichis en azote, comme les zones de pâturage des troupeaux d'herbivores. Leur présence constante dans les cinq coprolithes, mais également celle, plus ponctuelle, des Urticaceae, Lamiaceae, Brassicaceae, Ranunculaceae ou Chenopodiaceae, dont certaines espèces sont nitrophiles, pourraient mettre en évidence les zones de paquage des herbivores et donc le territoire de chasse des prédateurs (carnivores et hommes), à proximité du site. La surreprésentation de Carduus est probablement à relier au comportement du carnivore (léchage de patte ou de fourrure après contact avec des chardons sur son territoire) ou à un apport par un insecte pollinisateur juste après l'émission du fèces.

Les cinq coprolithes révèlent donc un paysage semiouvert de prairie ponctuée de zones humides et de bosquets de pins et d'arbres mésothermophiles, ces derniers étant plus représentés dans la base du remplissage. Ces données possèdent les caractéristiques de territoires de chasse des hyénidés. Dans les ensembles V et II, les données polliniques issues des sédiments (RenaultMiskovsky, 1972 ; Messouci, 1993) et des coprolithes semblent s'accorder. Parmi le cortège d'herbacées, les Poaceae sont plus représentées dans le sédiment que dans le coprolithe, mais le reste du cortège herbacé est relativement homogène, avec la présence des Cichorioideae.

\section{2 - DONNÉES PARASITOLOGIQUES}

Les analyses parasitologiques ont permis de montrer que certains animaux, probablement les hyènes, étaient touchés par des ascaridioses. Mais la quantité d'œufs identifiés ne permet pas de déterminer si la parasitose concernait la hyène. Il est plutôt envisageable qu'elle ait mangé une proie contaminée, ou une proie porteuse mais non contaminée, appelée " hôte paraténique », ou peutêtre un très jeune canidé. En effet, Toxocara sp. parasite les mammifères carnivores, comme les canidés et les hyénidés ( $T$. canis) et les félidés (T. cati, T. mystax). Toxocara sp. a un cycle de vie monoxène, c'est-à-dire à un seul hôte, dit définitif, sans hôte intermédiaire pour poursuivre sa maturation, qui se fait dans le milieu extérieur. Alors que $T$. cati peut résister à des températures comprises entre $-10^{\circ} \mathrm{C}$ et $45^{\circ} \mathrm{C}, T$. canis, quant à lui, nécessite des conditions climatiques plus spécifiques, avec de l'ombre, une humidité importante et des températures comprises entre 15 et $30^{\circ} \mathrm{C}$ (Dorchies \& Guitton, 1993). L'hôte se contamine en mangeant des hôtes paraténiques, comme 
les rongeurs, ou en mangeant de l'herbe souillée ou encore en se léchant après être entrés en contact avec de la végétation contaminée. Le ver adulte se loge dans l'intestin grêle de son hôte définitif et y pond jusqu'à 200000 œufs par jour. La contamination peut également avoir lieu chez les canidés in utero ou au moment de l'allaitement, tant chez les canidés que les félidés (Bourdeau, 1993). La présence de Toxocara a été mise en évidence dans des sédiments datés entre 300000 et 500 000, à MenezDregan (Bouchet et al., 2003), mais la présence de cette parasitose n'avait pas encore été montrée au Pléistocène supérieur dans le sud de la France.

Ascaris sp. a également un cycle monoxène et parasite de nombreux mammifères (canidés, félidés, suidés, équidés, bovidés, homme, etc.). La femelle pond jusqu'à 200000 œufs par jour, qui sont libérés dans le milieu extérieur. Après une phase de maturation, les œufs sont avalés par l'hôte, en se léchant ou en mangeant de la nourriture souillée ou contaminée (Bourdeau, 1993). Cette étude recule la date de présence d'Ascaris en France, le plus ancien œuf ayant été découvert dans la Grande Grotte d'Arcy-sur-Cure, dans des sédiments datés d'environ 30000 ans (Bouchet et al., 1996).

\section{6 - CONCLUSION}

Dans un contexte d'étude pluridisciplinaire, la reconstitution des paléoenvironnements végétaux est très importante lors de l'étude d'un site archéologique et des comportements humains fossiles. Cependant, les remplissages sédimentaires sont soumis à de nombreux phénomènes taphonomiques (oxydation, remaniement, etc.) aboutissant à la destruction partielle voire complète du matériel sporo-pollinique. Afin de compléter et/ou de valider les données polliniques, les coprolithes peuvent être une alternative pour obtenir des données sur l'environnement végétal, comme c'est le cas dans le site des Ramandils où l'analyse pollinique du remplissage n'avait permis d'obtenir des informations que sur les ensembles stratigraphique $\mathrm{V}$ et II. Elles sont bien sûr à prendre avec prudence mais constituent une source précieuse d'informations. Les données polliniques, issues de cinq coprolithes sur les douze qui ont été analysés, mis au jour dans les ensembles stratigraphiques V, II et I, a permis, d'une part, de confirmer et de préciser les données précédemment obtenues, et d'autre part, de compléter les données dans le sommet du remplissage, dont les sédiments détritiques n'avaient livré aucun résultat exploitable.

Ces données seront complétées par des analyses d'autres coprolithes et confrontées aux données des autres disciplines (grande faune et malacofaune notamment) afin d'affiner le contexte environnemental et climatique et de préciser le cadre chronologique du remplissage des Ramandils, au cours de cette période du Pléistocène supérieur.

Les analyses parasitologiques seront également poursuivies pour tenter de compléter le spectre des parasitoses. Bien que cette étude ait permis de montrer la présence d'Ascaris sp. et de Toxocara sp. à cette période du Pléistocène supérieur dans le sud de la France, d'autres espèces pourraient être découvertes. En effet, la hyène, dont les coprolithes sont les mieux conservés, est traditionnellement parasitée par Toxocara canis, mais également par Taenia crocutae, Diphyllobothrium sp., Toxascaris sp., Cyclicospirura sp. et Dipylidium sp. (Ferreira et al., 1993), dont les présences pourraient être démontrées.

\section{RÉFÉRENCES BIBLIOGRAPHIQUES}

ARGANT J., 1990 - Climat et environnement au Quaternaire dans le Bassin du Rhône d'après les données palynologiques. Documents des Laboratoires de Géologie, Lyon, 111, 1-199.

ARGANT J., 2010 - Palynologie des coprolithes d'hyène de Romainla-Roche (Doubs, France) : apport paléoenvironnemental. Revue de Paléobiologie, Genève, 29 (2), 473-476.

ARGANT J., \& BONIFAY M.F., 2011 - Les coprolithes de hyène (Pachycrocuta brevirostris) de la couche 2 du site villafranchien de Ceyssaguet (Lavoûte-sur-Loire, Haute-Loire, France) : analyse pollinique et indications paléoenvironnementales). Quaternaire, 22 (1), 3-11

ARGANT J., \& PHILIPPE M., 2011 - L'analyse pollinique de coprolithes : un outil pour la reconstitution du paléoenvironnement. Quaternaire, Hors-Série 4, 307-318.

BANES L., \& DORIGNY, A., 2005 - Le gisement des Ramandils (Port-La Nouvelle, Aude). Approche paléoenvironnementale. In Tuffreau, A. (Eds.), Peuplements humains et variations environnementales au Quaternaire, Colloque de Poitiers (18-20 septembre 2000). BAR International Series 1352, Oxford, 95-104.

BOUCHET F., 2001 - La paléoparasitologie. In J.-C. Miskovsky (dir.) Géologie de la Préhistoire : méthodes, techniques, applications. Géopré, Perpignan, 911-918.

BOUCHET F., \& BOULARD Y., 1991 - Ultrastructural changes following treatment with a micro-wave pulse in an oocyst of Eimeria magna Pérard, 1925. Parasitology Research, 77 (7), 585-589.

BOUCHET F., BAFFIER D., GIRARD M., MOREL P., PAICHELER J.C., \& DAVID F., 1996 - Palaeoparasitology in a Pleistocene context: initial observations in the Grande Grotte at Arcy-sur-Cure (Department of the Yonne, France). Compte-Rendu de l'Académie des Sciences, Série III, 319, 147-151

BOUCHET F., ARAÚJO A., HARTER S., MIRANDA CHAVES S, DUARTE A.N., MONNIER J.L., \& FERREIRA L.F., 2003 - Toxocara canis (Werner, 1782) eggs in the Pleistocene Site of MenezDregan, France (300,000-500,000 Years Before Present). MemÓrias do Instituto Oswaldo Cruz, Rio de Janeiro, 98 (Suppl. 1), 137-139.

BOULBES N., \& RUSCH L., 2016 - Contribution à l'étude des Equidés : les restes de chevaux du site des Ramandils (Port-laNouvelle, Aude). In T. Saos (dir.) Le Monde moustérien méditerranéen entre Rhône et Pyrénées. Rapport 2016 du Programme Collectif de Recherches. Bilan triennal 2014-2016, S.R.A. de Montpellier, 143-152.

BOURDEAU P., 1993 - Ascaridiose des carnivores. Encyclopédie vétérinaire - Parasitologie, 5, 0100, 1-10.

BOUTIÉ P., AJAJA O., BANÈS L., MOLÈS V., KABIRI L., \& GRÉGOIRE S., 1994 - Le gisement moustérien des Ramandils (Port-la-Nouvelle, Aude), Rapport de fouille programmée, (saisons 1992-1994). Archéologie de la France-Informations, (http://adlfi. revues.org/11756).

BUCKLAND W., 1829 - On discovery of coprolites, or fossils feces, in the Lias at Lyme Regis, and in other formations. Geological Society of London, Transactions Series, II, 3, 223-236.

CALLEN E.O., \& CAMERON T.W.M., 1960 - A prehistoric diet revealed in coprolites. The New Scientist, 90, 35-40.

CARRION J.S., SCOTT L., HUFFMAN T., \& DREYER C., 2000 - Pollen analysis of Iron Age cow dung in southern Africa. Vegetation History and Archaeobotany, 9 (4), 239-249.

CARRION J.S., SCOTT L., ARRIBAS A., FUENTES N., GILROERA G., \& MONTOYA E., 2007 - Pleistocene landscapes in central Iberia inferred from pollen analysis of hyena coprolites. Journal of Quaternary Science, 22 (2), 191-202.

DORCHIES P., \& GUITTON C., 1993 - Les ascaridioses des carnivores domestiques. Recueil de Médecine vétérinaire, $169(5 / 6)$ 333-343. 
FERREIRA L.F., ARAUJO A., \& DUARTE AN., 1993 - Nematode larvae in fossilized animal coprolites from Lower and Middle Pleistocene sites, Central Italy. Journal of Parasitoly, 79 (3), 440-442.

FLORENZANO A., MARIGNANI M., ROSATI L., FASCETTI S., \& MERCURI A.M., 2015 - Are Cichorieae an indicator of open habitats and pastoralism in current vegetation in southern Italy? A test to guide the pollen interpretation in palaeobotanical data. Plant Biosystems, 149 (1), 154-165.

FUGASSA M.H., BELTRAME M.O., SARDELLA N.H., CIVALERO M.T., \& ASCHERO C., 2009 - Paleoparasitological results from coprolites dated at the Pleistocene-Holocene transition as source of paleoecological evidence in Patagonia. Journal of archaeological Science, 37 (4), 880-884.

GATTA M., SINOPOLI G., GIARDINI M., GIACCO B., HADJAS I., PANDOLFI L., BAILEY G., SPIKINS P., ROLFO M.F., \& SADORI L., 2016 - Pollen from Late Pleistocene hyena (Crocula crocuta spelea) coprolites: an interdisciplinarity approach from two italian sites. Review of Palaeobotany and Palynology, 233, 56-66.

GONZALES-SAMPERIZ P., MONTES L., \& UTRILLA P., 2003 - Pollen in hyena coprolites from Gabasa Cave (northern Spain). Review of Palaeobotany and Palynology, 126 (1-2), 7-15.

HARTER S., LAILHEUGUE S., LE MORT F., VIGNE J.D., GUILAINE J., LE BRUN A., \& BOUCHET F., 2005 - Premières données parasitologiques sur les populations humaines précéramiques chypriotes (VIII et $\mathrm{VII}^{\mathrm{e}}$ millénaires av. J.-C.). Paléorient, 31 (2), 43-54.

JOUY-AVANTIN F., 1997 - Diversité des paléoparasites dans le monde. Etude paléoparasitologique préliminaire du site pléistocène moyen de la Caune de l'Arago. DEA, Museum National d'Histoire Naturelle, Université de Perpignan Via Domitia, Perpignan, 112 p.

JOUY-AVANTIN F., 2004 - Paléoparasitologie : Contribution à l'étude des paléoenvironnements de sites pléistocènes et holocènes du littoral méditerranée français. Thèse de l'Université de Perpignan Via Domitia, Perpignan, $552 \mathrm{p}$.

JOUY-AVANTIN F., COMBES C., LUMLEY (de) H., MISKOVSKY J.C., \& MONÉ H., 1999 - Helminth eggs in animal coprolites from a Middle Pleistocene site in Europe. Journal of Parasitology, 85 (2), 376-379.

JOUY-AVANTIN F., DEBENATH A., MOIGNE A.M., \& MONE H., 2003 - A standardized Method for the description and the study of coprolites. Journal of Archaeological Science, 30 (3), 367-372.

LARKIN N., ALEXANDER J., \& LEWIS M., 2000 - Using experimental studies of recent faecal material to examine hyaena coprolites from the West Runton Freshwater Bed, Norfolk, England. Journal of Archaeological Science, 27 (1), 19-31.

LEBAILLY M., HARTER S., \& BOUCHET F., 2003 - La paléoparasitologie, à l'interface de l'archéologie et de la biologie. Archéopages, 11, 12-17.

LEROI-GOURHAN A., 1966 - L'analyse pollinique des coprolithes. Bulletin de la Société Préhistorique Française, 63 (5), 163-164.
MARTILL D.M., 1994 - La fossilisation instantanée. La Recherche, 269, 996-1002.

MARTIN PS., \& SHARROCK F.W., 1964 - Pollen Analysis of Prehistoric Human Faeces: A New Approach to Ethnobotany. American Antiquity, 30 (2), 168-180.

MESSOUCI S., 1993 - Contribution à l'étude palynologique du site moustérien des Ramandils. Mémoire de DEA du MNHN Paris VI, Paris, $48 \mathrm{p}$.

MOLES V., 2008 - Étude techno-économique et typologique des industries de la grotte des Ramandils (Port-la-Nouvelle, Aude, France). Contribution à la reconnaissance d'une microproduction au Paléolithique moyen. Thèse de Doctorat, Université Paul Valéry, Montpellier III, Montpellier, 375 p.

RENAULT-MISKOVSKY J., 1972 - Contribution à la paléoclimatologie du Midi méditerranéen pendant la dernière glaciation et le post-glaciaire, d'après l'étude palynologique du remplissage des grottes et abris-sous roche. Thèse de Doctorat d'Etat, Paris VI, Paris, $495 \mathrm{p}$.

RUSCH L., 2015 - La faune de la grotte des Ramandils (Portla-Nouvelle, Aude). Taphonomie et archéozoologie des grands mammifères de l'ensemble III. Mémoire de Master Préhistoire et Paléoenvironnements quaternaires, $2^{\mathrm{e}}$ année, Université de Perpignan Via Domitia, Perpignan, 60 p.

SCOTT L., 1987 - Pollen analysis of hyena coprolites and sediments from Equus Cave, Taung, southern Kalahari (South Africa). Quaternary research, 28 (1), 144-156.

SCOTT L., \& BRINK J.S., 1992 - Quaternary palaeoenvironment of pans in central South Africa: palynological and palaeontological evidence. South African Geographer, 19, 22-35.

SCOTT L., FERNANDEZ-JALVO Y., CARRION J.S., \& BRINK J., 2003 - Preservation and interpretation of pollen in hyena coprolites: taphonomic observations from Spain and southern Africa. Palaeontologia Africana, 39, 83-91.

VAN CLEAVE H.J., \& ROSS J.A., 1947 - A method for reclaiming dried zoological specimens. Science, 105 (2725), 318.

VIVENT D., 1989 - Analyses polliniques de coprolithes d'hyènes de deux sites paléolithiques d'Auvergne (Saint-Hippolyte, Puy-deDôme et Châtelperron, Allier). Revue archéologique du Centre de la France, 28 (2), 229-235.

WHARTON D.A., 1980 - Nematode egg-shells, Parasitology, 81 (2), 447-463.

YLL R., CARRION J.S., MARRA A.C., \& BONFIGLIO L., 2006 - Vegetation reconstruction on the basis of pollen in Late Pleistocene hyena coprolites from San Teodoro Cave (Sicily, Italy). Palaeogeography, Palaeoclimatology, Palaeoecology, 237 (1), 32-39.

YOKOYAMA Y., NGUYEN H.V., SHEN G., QUAEGEBEUR J.P., \& KOUMBA N., 1987 - Datation par les méthodes des familles de l'Uranium et la résonnance de spin électronique (ESR) du remplissage de la grotte des Ramandils à Port-la-Nouvelle (Aude, France). Cypsela, 6, 151-154. 